\title{
PREPARATION OF VERY LARGE YBCO FILMS ON SINGLE- AND POLYCRYSTALLINE SUBSTRATES
}

\author{
H. Kinder, W. Prusseit, R. Semerad and B. UtZ
}

Technical University of München, Physics Department E 10, 85747 Garching, Germany

High quality $\mathrm{YBa}_{2} \mathrm{Cu}_{3} \mathrm{O}_{7}$ thin films on areas of up to $9^{\prime \prime}$ in diameter have been grown by reactive thermal co-evaporation using a rotating substrate holder. A scanning inductive $j_{c}$ probe is used to monitor the homogeneity of critical current densities which are typically greater than $2 \mathrm{MA} / \mathrm{cm}^{2}$. The same technique also allows a double sided deposition for filter applications. Surface resistance values are found to be below $500 \mu \Omega$ at $10 \mathrm{GHz}$. On biaxially aligned buffer layers on polycrystalline substrates $j_{c}$ values of 1.4 MA/ $\mathrm{cm}^{2}$ are achieved at $77 \mathrm{~K}$ as well. We have also studied the properties of $\mathrm{Nd}_{1-x} \mathrm{Ba}_{2+x} \mathrm{Cu}_{3} \mathrm{O}_{7}$ films grown with the same system.

PACS numbers: $81.15 . \mathrm{Cd}, 74.72 .-\mathrm{h}, 68.55 .-\mathrm{a}$

\section{Introduction}

Applications of high temperature superconductors based on thin films are considered to be among the most promising ones to date. Rapid progress has been made particularly on RF devices such as high order multipole filters for cellular or satellite communications, low noise NMR pickup antennas, and radar delay lines. These activities triggered a huge demand for large size HTSC thin film material mainly on single crystal substrates. Aside from the thallium cuprates, the material of choice to be $\mathrm{YBa}_{2} \mathrm{Cu}_{3} \mathrm{O}_{7}$ ( $\mathrm{YBCO}$ ).

In addition, more and more attention is paid to high power applications as well. On the one hand, there is a considerable effort to produce long HTSC coated conductors for power transmission or for magnets, on the other hand, films on very large substrates will be used as resistive fault current limiters. Both applications require thick YBCO films deposited on very large substrates which are available only in polycrystalline form, as metal tapes or ceramic materials.

These potential applications basically rely on the availability of reasonably priced large area HTSC thin film material. Many deposition techniques including co-evaporation [1], sputtering, pulsed laser deposition (PLD) and metal-organic chemical vapor deposition (MOCVD) have shown their capability to deposit high quality YBCO films on single crystal substrates up to $4^{\prime \prime}$ diameter. Nevertheless, for a commercial use cost effectiveness and thus a production at a high volume 
deposition rate are crucial. PLD and co-evaporation can deposit with the highest rates limited only by the growth process itself, while MOCVD, sputtering and co-evaporation can be realized on the largest areas. Obviously co-evaporation combines both advantages and thus seems to be a promising choice for commercial scale thin film production.

To utilize the advantages of this technique we graded up our existing co-evaporation system and further increased the area of deposition to a diameter of $9^{\prime \prime}$ or $20 \mathrm{~cm}$. We grow YBCO films on a variety of single crystal substrates as well as on polycrystalline samples.

\section{Thermal co-evaporation using a heater with an oxygen pocket}

A sketch of the deposition setup is shown in Fig. 1. The metals $\mathrm{Y}, \mathrm{Ba}$ and $\mathrm{Cu}$ are evaporated from resistively heated boats which are mounted in a vacuum chamber with a base pressure below $2 \times 10^{-6}$ mbar. The evaporation rate of each metal is controlled by an individual quartz crystal monitor with a collimator tube in front of it to confine its sight to only one boat. The rates have to be controlled to approximately $1 \%$ accuracy to avoid outgrowth and to get high quality films reproducibly.

For the formation of YBCO films an oxygen partial pressure of approximately $10^{-3}$ mbar is needed. At the same time the chamber pressure must be below

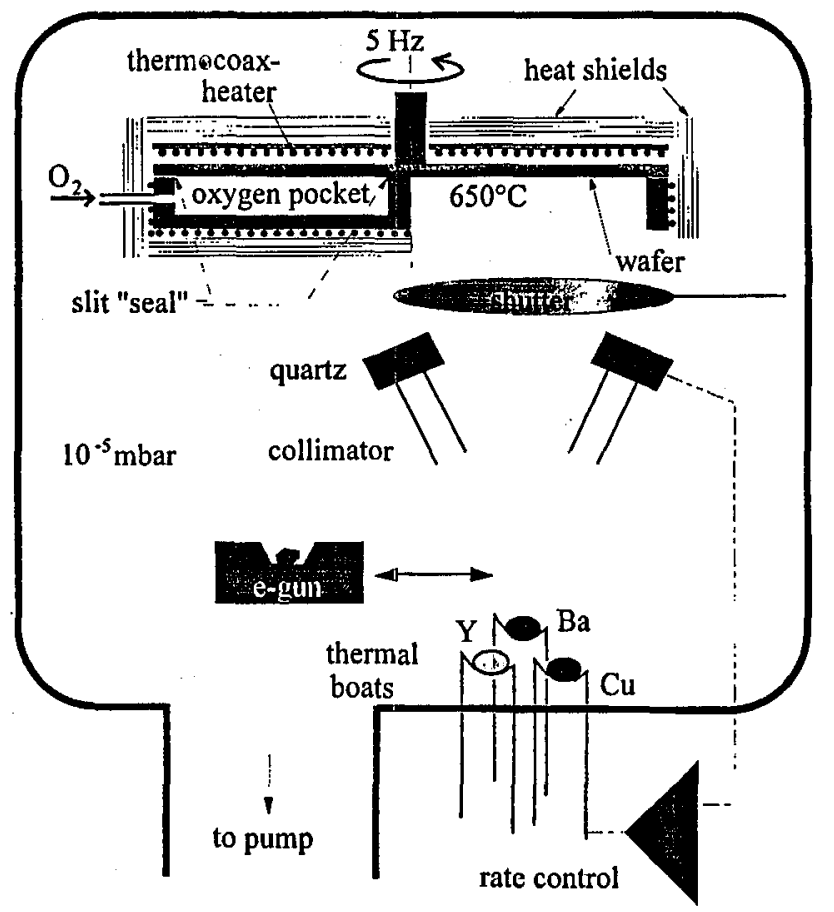

Fig. 1. Deposition scheme for thermal co-evaporation combined with an oxygen pocket heater. 
$10^{-5} \mathrm{mbar}$ at least to ensure that the metal vapor can reach the substrate nearly ballistically from a large distance. To meet both requirements the substrates are placed on a rotating disk and alternated between a zone of the metal deposition on the one side and an oxygen pocket on the other. At a rotation frequency greater than 5 the metal atoms from the vapor condense on the substrates and oxidize consecutively in the pocket forming YBCO. The oxygen is effectively sealed from the vapor zone by keeping the slit between the pocket and the rotating disk very narrow (width typically $0.3 \mathrm{~mm}$ ). An oxygen partial pressure drop greater than 1:100 is easily achieved. The substrate is heated radiatively from the rear, the sides and from the covered part of the bottom by thermocoax heater wire to a temperature of $650^{\circ} \mathrm{C}$. YBCO films are deposited with a rate of typically $24 \mathrm{~nm} / \mathrm{min}$. The film thickness generally is in the range of $300 \mathrm{~nm}$ to $2 \mu \mathrm{m}$.

To adjust the ratio of the evaporation rates we analyzed the film composition with a heavy ion Rutherford scattering (RBS). Once the stoichiometry has been fixed, the process is stable on a months time scale. Small variations in composition allow us to optimize the films, e.g. in respect to high $T_{\mathrm{c}}$ or very smooth surfaces. These small deviations can be easily monitored by changes in the surface morphology as observed in high resolution scanning electron microscopy (SEM) images.

A fourth boat next to the other ones is used to cap YBCO films with in situ gold overlayers to achieve good contact resistances below $10^{-8} \Omega \mathrm{cm}^{2}$ which are desired for contacts in power- and RF-applications. In addition oxide buffer layers like $\mathrm{MgO}, \mathrm{CeO}_{2}$ or $\mathrm{YSZ}$ can be deposited in situ by an e-beam gun which can be moved above the boats, see Fig. 1. This system allows us to deposit YBCO film on a variety of substrates including $\mathrm{MgO}, \mathrm{YSZ}, \mathrm{LaAlO}_{3}, \mathrm{CeO}_{2}$ buffered sapphire, YSZ buffered $\mathrm{Si}$ and even MgO buffered GaAs.

The heater is designed for $9^{\prime \prime}$ diameter sample trays. This area can be flexibly filled either with one single $8^{\prime \prime}$ wafer or higher numbers of smaller samples. Insets for square shaped substrates accompanied by small substrates to monitor the deposition success can be used as well (Fig. 2).
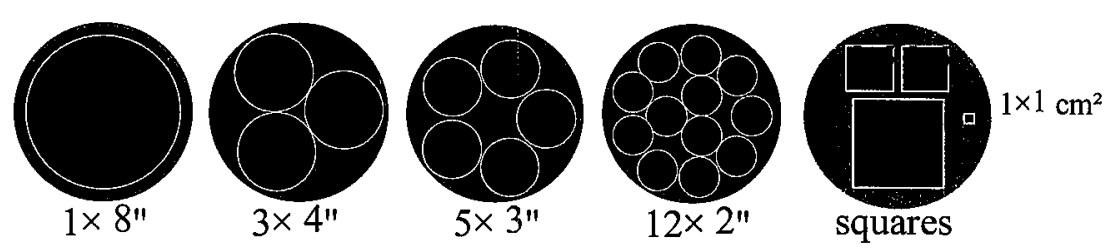

Fig. 2. Various insets for the $9^{\prime \prime}$ heater: Depending on the substrate size larger quantities of substrates can be coated in the heater simultaneously.

During the deposition the rear of the substrates is exposed only to vacuum which does no-harm. Consequently this allows us to produce double sided films by simply flipping the substrates over ex situ after a first deposition step and fabricating another film on the backside of the wafer without degrading the first one. 


\section{Results on single crystal substrates}

Using the sample inset for $12 \times 2^{\prime \prime}$ wafers, $T_{\mathrm{c}}$ of $450 \mathrm{~nm}$ thick YBCO films has been measured on wafers both from the inner circle and the outer circle of the tray. We find $T_{c}$ values in the range of 88.4 to $88.8 \mathrm{~K}$, homogeneous over the heater radius (Fig. 3).

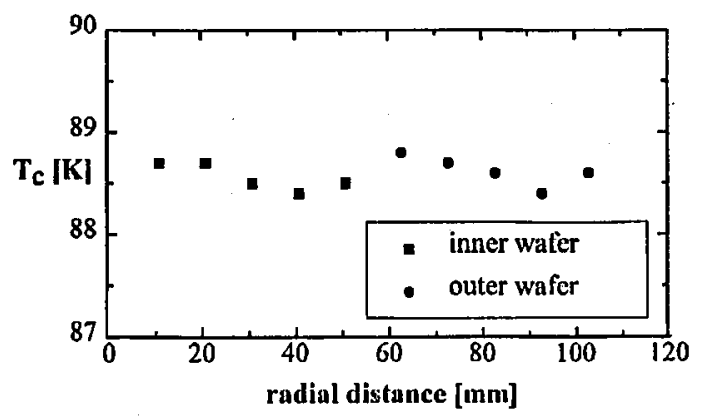

Fig. 3. $T_{c}$ distribution over the heater radius, measured on two $2^{\prime \prime} \mathrm{LaAlO}_{3}$ wafers.

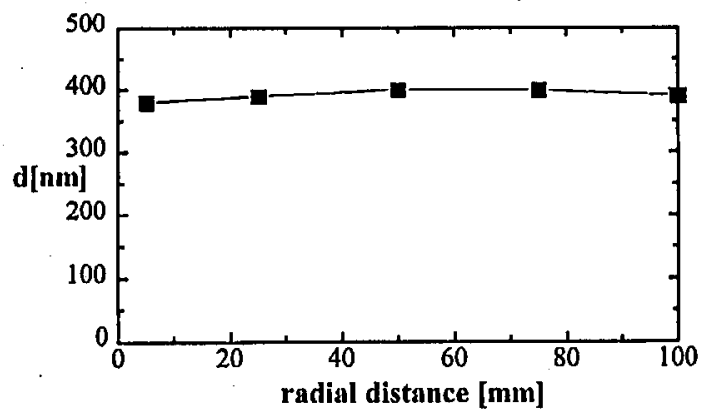

Fig. 4. Film thickness $d$ over the heater radius of $4^{\prime \prime}$ or $100 \mathrm{~mm}$.

A large source to substrate distance of about $35 \mathrm{~cm}$ in combination with the wide vapor cone intrinsic to evaporation sources results in a good thickness homogeneity. A variation of smaller than $3 \%$ has been confirmed by a set of small samples placed on the radius (Fig. 4).

The critical current density is routinely mapped by an apparatus scanning a set of coils over the film surface and measuring $j_{\mathrm{c}}(77 \mathrm{~K})$ inductively [2]. The resulting $j_{\mathrm{c}}$ maps show clearly that the critical current density is extraordinarily homogeneous on the whole area of a $4^{\prime \prime}$. wafer. Moreover, there is no significant difference between the first and the second side of double sided YBCO films (Fig. 5).

This holds also for the surface resistance $R_{\mathrm{s}}$ : the films on both sides show typical $R_{\mathrm{s}}$ values below $500 \mu \Omega$ at $10 \mathrm{GHz}$ as has been measured by a dielectric resonator setup. 


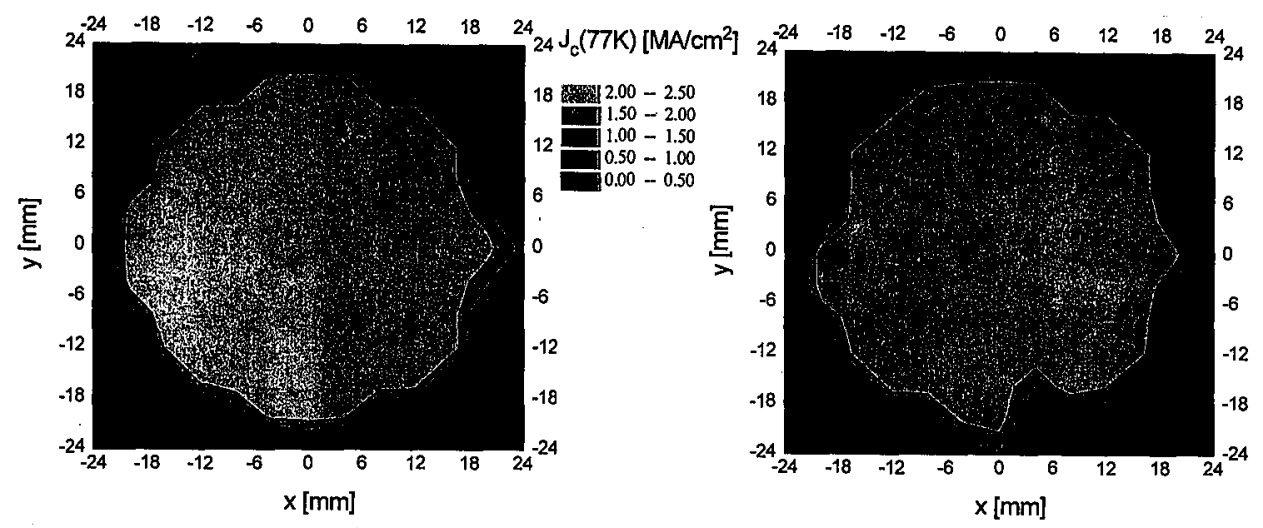

Fig. 5. $j_{c}(77 \mathrm{~K})$ maps of YBCO films of a double sided $2 " \mathrm{LaAlO}_{3}$ wafer showing that both sides have equally good superconducting properties and homogeneity.

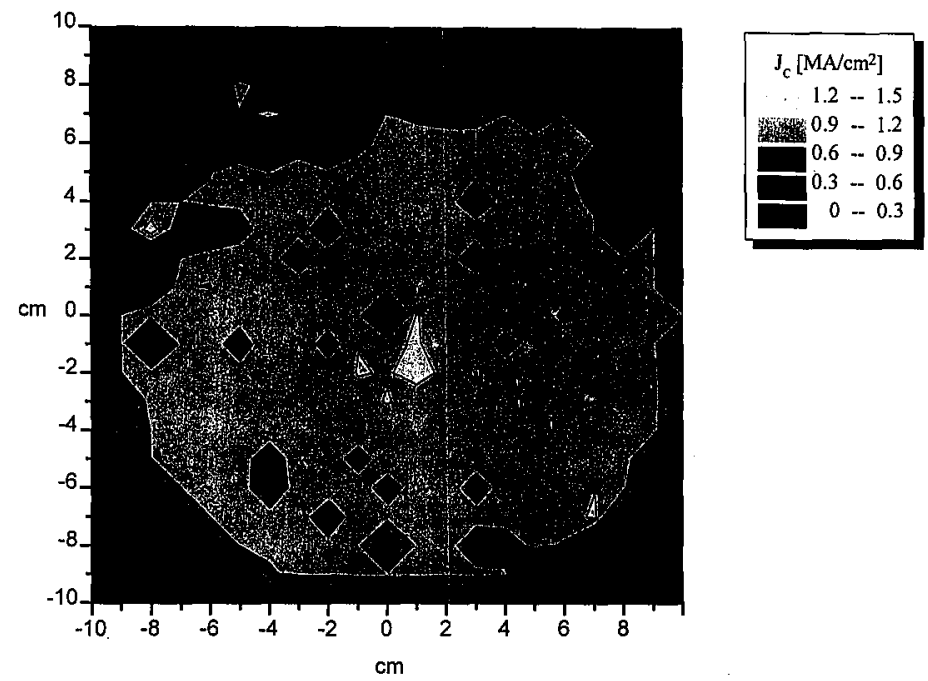

Fig. 6. $j_{\mathrm{c}}(77 \mathrm{~K})$-map of $8^{\prime \prime}(20 \mathrm{~cm})$ film on sapphire after being kept on air for one year.

For demonstration purposes we have also coated an $8^{\prime \prime}$ sapphire wafer by YBCO. To map out its $j_{\mathrm{c}}$ we had to build a larger scanner which was not ready before the film was one year old and had been travelling around the world to various conferences. Figure 6 shows that the film was still quite good even after this history.

\section{Deposition and properties of Nd-123 films}

In the search for materials with superior microwaver surface resistance $\left(R_{\mathbf{s}}\right)$ within the 123-family, neodymium instead of yttrium seems to be a promising candidate. With increasing ionic radius the rare earth constituent can occupy Ba-sites 


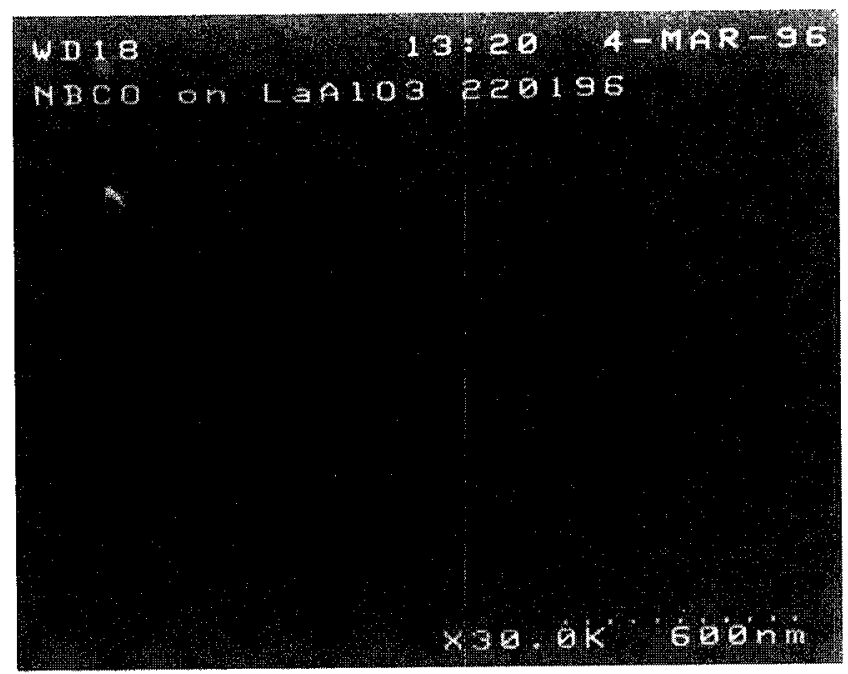

Fig. 7. Scanning electron micrograph of a $\mathrm{Nd}_{1+x} \mathrm{Ba}_{2-x} \mathrm{Cu}_{3} \mathrm{O}_{7}$ film with $x=0.18$. It is remarkably smooth.

and the transition temperature can be raised up to $95 \mathrm{~K}$ [3]. Consequently, an improved microwave performance may be expected for operating temperatures in the range between $60 \mathrm{~K}$ and $80 \mathrm{~K}$, typically maintained in ITSC cellular base station filters. Therefore, we replaced the $\mathrm{Y}$ in one of the boats by $\mathrm{Nd}$ and performed a growth study of $\mathrm{Nd}_{1+x} \mathrm{Ba}_{2-x} \mathrm{Cu}_{3} \mathrm{O}_{7}$ (NBCO) films.

By a systematic change of the $\mathrm{Nd} / \mathrm{Ba}$ flux ratio the $\mathrm{Nd}_{1+x} \mathrm{Ba}_{2-x} \mathrm{Cu}_{3} \mathrm{O}_{7}$ composition has been varied from the Nd-rich $(x=0.18)$ to the Ba-rich side $(x=-0.10)$. For the ideal stoichiometry $(x \approx 0)$ we achieved sharp transitions above $93 \mathrm{~K}$ and $j_{c}(77 \mathrm{~K})=2 \div 4 \mathrm{MA} / \mathrm{cm}^{2}$. On the Nd-rich side the films grow remarkably smooth. Figure 7 depicts an SEM image of a NBCO film with $x=0.18$. Obviously, there is no resolvable surface structure. Only atomic force microscopy reveals shallow terraces constituting a surface roughness of $50 \AA$.

\section{YBCO films on polycrystalline substrates with biaxially aligned buffer layers}

Up to now most YBCO thin film work had to be done on single crystal substrates to get reasonably good superconducting properties. Nevertheless, the size of substrates available as single crystals generally is limited to a few inches in diameter which might not be sufficient for applications like resistive fault current limiters based on thin films. Larger substrates are available only in a polycrystalline form.

It has been shown recently that good electrical properties of YBCO thin films can also be achieved on polycrystalline metal substrates with biaxially textured buffers produced by ion beam assisted deposition (IBAD) [4, 5]. For fault current limiters, however, these buffers have to be fabricated on isolating substrate material. An excellent texture of YSZ buffer layers recently has been achieved on 
polycrystalline YSZ ceramics [6] with a similar IBAD technique. We deposited YBCO layers on buffered substrates of this group with the evaporation system described above.

YBCO films $700 \mathrm{~nm}$ to $1.4 \mu \mathrm{m}$ thick have been deposited on small poly-YSZ substrates with an IBAD buffer using the same parameters as for single crystal substrates. We measured a $T_{\mathrm{c}}>88 \mathrm{~K}$ and $j_{\mathrm{c}}$ values around $1 \mathrm{MA} / \mathrm{cm}^{2}$ inductively. The best critical current density was achieved on a YSZ buffer $800 \mathrm{~nm}$ thick resulting in $j_{c}=1.4 \mathrm{MA} / \mathrm{cm}^{2}$ as has been confirmed by a resistive measurement.

IBAD buffers are now available even on larger YSZ ceramics with an area of $5 \times 5 \mathrm{~cm}^{2}$ and $10 \times 10 \mathrm{~cm}^{2}$. YBCO films deposited on these substrates show a $T_{\mathrm{c}}>87 \mathrm{~K}$. The critical current density has been mapped by the scanning $j_{\mathrm{c}}$ probe mentioned above. Although there are still some inhomogeneities at the edges of the substrate, it can clearly be seen that $j_{c}>0.7 \mathrm{MA} / \mathrm{cm}^{2}$ has been reached quite homogeneously on most of the substrate area (Fig. 8).

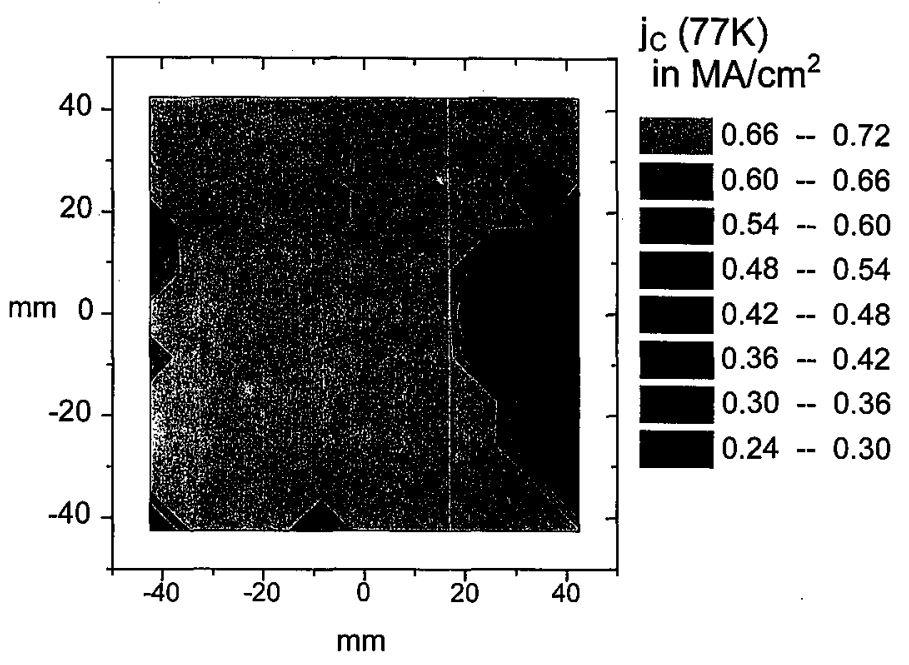

Fig. 8. $j_{c}$ map of $1.4 \mu \mathrm{m}$ YBCO on a $900 \mathrm{~nm}$ YSZ buffer biaxially aligned by IBAD on polycrystalline YSZ ceramics.

The in-plane orientation of YSZ varies between $13.8^{\circ}$ in the center and $18.3^{\circ}$ in the edges while the YBCO in-plane texture changes from $6.35^{\circ}$ to $8.25^{\circ}$.

\section{Conclusions}

We have shown that thermal co-evaporation can produce YBCO films on areas of $9^{\prime \prime}$ diameter with excellent homogeneity. The process has a good long-time stability and good reproducibility while the yield approaches $100 \%$. The cost of this process is reduced due to a volume deposition rate which is the highest of all thin film techniques currently used for YBCO film production.

Substrates of every size and shape up to $8^{\prime \prime}$ diameter or $16 \mathrm{~cm}$ side length can be coated with the system. Good superconducting properties of YBCO have been achieved not only on single crystal substrates. On biaxially aligned buffers on 
polycrystalline material we got very high critical current densities on large areas which is unique worldwide to our knowledge.

We produced NBCO films by simply replacing the rare earth element. The films had excellent quality and an outstanding smooth surface morphology. This gives a hint that films of the whole class of rare earth HTSC compounds can be easily fabricated by thermal co-evaporation. Thermal co-evaporation seems to be the technique of choice to produce the large quantities of large area YBCO films that might be demanded for applications in the near future.

\section{References}

[1] P. Berberich, B. Utz, W. Prusseit, H. Kinder, Physica C 219, 497 (1994).

[2] H. Kinder, P. Berberich, B. Utz, W. Prusseit, IEEE Trans. Appl. Supercond. 5, 1575 (1995).

[3] G.V.M. Williams, J.T. Tallon, Physica $C$ 258, 41 (1996).

[4] Y. Iijima, N. Tanabe, O. Kohno, Y. Ikeno, Appl. Phys. Lett. 65, 769 (1992).

[5] X.D. Wu, S.R. Foltyn, P.N. Arendt, W.R. Blumenthal, I.II. Campbell, J.D. Cotton, J.Y. Coulter, W.L. Hults, M.P. Maley, H.F. Safar, J.L. Smith, Appl. Phys. Lett.67, 2397 (1995).

[6] J. Wiesmann, K. Heinemann, II.C. Freyhard, IEEE Trans. Appl. Supercond. (ASC96 proceedings), in print. 